\title{
INTRODUCTION TO THE SPECIAL ISSUE ON IOT CLOUD SOLUTIONS FOR SOCIETAL APPLICATIONS
}

Addressing societal problems such as air pollution, water contamination, corruption, healthcare management, agricultural assistance, and so forth has increased in the recent past using several highend technologies, including IoT cloud.

In fact, innovations have become the key factor of economic growth in several developing and developed countries. For instance, Atal Innovation Mission of India has promoted innovations that addresses the needs of the society through IoT cloud based technologies at AIC-IIITKottayam; TUM-Germany and several top ranked universities across the globe have driven the research or product developments targeting the benefits of the society energy, healthcare, agriculture, economic health, and so forth.

Existing IoT based societal applications require a large volume of data for the analysis; a secure environment for handling data (either in the cloud or edge environments); and, a diligent planning while handling heterogeneous devices on federated cloud environments. In fact, scalability of cloud resources is the backbone for IoT cloud environments, while edge computing is required for short response times. Many IoT applications are based on automatic decision making applying machine learning on huge data sets and thus, these techniques influenced a large group of researchers in the IoT cloud domain.

This special issue on IoT Cloud Solutions for Societal Applications discusses security aspects and a decision making in IoT applications. Syed et al discuss IoT security requirements, challenges, and in the context of smart cities, smart health, smart building, smart transport, and smart industry applications; Naveen et al have carried out a theoretical evaluation using spacio-temporal distance networks in a deep learning network in order to perform the action recognition tasks in IoT environments.

Shajulin Benedict, Indian Institute of Information Technology Kottayam, Kerala, India Michael Gerndt, Technische Universitaet Muenchen, Germany 